\title{
Transmission héréditaire de la résistance aux nématodes Meloidogyne Chitwood (Tylenchida) portée par 2 lignées de Capsicum annuum L. : étude de descendances homozygotes issues d'androgenèse
}

\author{
Hassan HENDY $\left({ }^{1}\right)$, Edmond POCHARD $\left({ }^{*}\right)$ \& Antoine DALMASSO \\ avec la collaboration technique de Michel BONGIOVANNI \\ I.N.R.A., Station de Recherches sur les Nématodes, 123, Boulevard Francis-Meilland, F 06602 Antibes \\ (*) I.N.R.A., Station d'Amélioration des Plantes maraîchères, Centre de Recherches d'Avignon, F 84140 \\ Montfavet
}

La lignée PM 217 de Capsicum annuum est résistante à Meloidogyne arenaria, $M$. incognita, $M$. javanica et $M$. sp. (Séville). La lignée PM 687 est résistante aux 3 premières espèces. Le parent-hôte opposé à chacune d'elles, "Yolo-Wonder », présente une résistance à $M$. javanica. Par androgenèse in vitro, on a obtenu des plantes haplö̈des à partir des hybrides $\mathrm{F}_{1}:$ PM $217 \times$ «Yolo Wonder » et PM $687 \times$ «Yolo-Wonder ». Chaque descendance autodiploìde a été soumise séparément à l'action de 5 populations-clones de Meloidogyne appartenant aux 4 espèces.

Il apparaît que PM 217 porte 2 gènes distincts et, vraisemblablement, indépendants, l'un contrôlant la résistance à $M$. arenaria, $M$. incognita et $M$. javanica, l'autre contrôlant la résistance à $M$. javanica et $M$. sp. (Séville). PM 687 porte également 2 gènes, l'un actif sur les 3 principales espèces, à l'exception d'une souche de $\boldsymbol{M}$. arenaria, l'autre contrôlant cette même souche. Ces 2 gènes sont probablement liés.

Ces divers gènes sont distincts par leur spectre d'action et/ou leur mode d'expression au niveau des racines. Les gènes à spectre le plus large, portés par chacun des géniteurs résistants, ne sont pas allèles, mais paraissent liés au même chromosome. "Yolo Wonder " porterait un $5^{\mathrm{e}}$ gène à effet plus limité.

Mots clés additionnels : Lignées autodiploïdes, piment, Solanaceae. L. : study of homozygous progenies obtained by androgenesis.

Capsicum annuum PM 217 was found resistant to Meloidogyne arenaria, M. incognita, M. javanica and $M$. sp. ( (Seville » type). Line PM 687 was resistant to the first three species. The host cultivar "Yolo Wonder", which was crossed with both lines, showed some resistance to $M$. javanica. Haploid plants were obtained by androgenesis trom $F_{1}$ hybrids PM $217 \times$ «Yolo Wonder" and PM $687 \times$ «Yolo Wonder». Each autodiploid progeny was separately inoculated with 5 clonal populations of Meloidogyne belonging to the 4 species. Two different and probably independent genes, one controlling resistance to $M$. arenaria, $M$. incognita, $M$. javanica, the other to $M$. javanica and $M$. sp. "Seville ", were demonstrated in PM 217 . Two genes were also observed in PM 687, one active against the 3 main species, except one $M$. arenaria population, the second controlling just this population. The 2 genes are probably linked. These genes differed by their range of effectiveness with regard to the Meloidogyne species and clones and/or by their expression in the roots. The broad spectrum genes from PM 217 and PM 687 respectively were not alleles but seemed to be located on the same chromosome. A fifth gene was observed in "Yolo Wonder" but with a very limited range of activity.

Additional key words : Autodiploid lines, sweet pepper, Solanaceae. 


\section{INTRODUCTION}

La recherche de sources de résistance aux nématodes à galles a été entreprise depuis longtemps chez le piment cultivé (HARE, 1956). Cet auteur a montré l'existence d'un gène dominant $\mathrm{N}$ dans le matériel «Santanka X S ». Ce gène contrôle la résistance à 3 espèces de Meloidogyne, mais avec une efficacité variable en fonction des souches et de la quantité d'inoculum (HARE, 1957). Le gène N a été incorporé à diverses variétés agronomiques, mais ce matériel ne semble pas être largement utilisé, sans doute à cause de sa résistance insuffisante, voire nulle, vis-à-vis de certaines populations de nématodes (DI VITO \& SACCARDO, 1978).

Nous avons récemment identifié 2 nouvelles sources de résistance efficaces vis-à-vis de populations très diverses appartenant aux 3 principales espèces de Meloidogyne : $M$. arenaria (Neal) Chitwood, $M$. incognita (Kofoid \& White) Chitwood et $M$. javanica (Treub) Chitwood (HENDY et al., 1983). L'une de ces sources est également résistante à une $4^{\mathrm{e}}$ espèce (souche «Séville »), espèce nouvelle en cours de description.

Pour identifier les gènes responsables de ces propriétés de résistance, les méthodes classiques d'analyse des générations de disjonction ne conviennent pas dans la mesure où ces gènes sont relativement nombreux et ont des champs d'activité qui se recouvrent partiellement. C'est essentiellement par leur spectre d'activité caractéristique à l'égard d'une gamme étendue d'espèces et de souches de nématodes que l'on peut espérer les révéler. Ceci suppose que le matériel en disjonction se présente sous forme de clones ou, mieux encore, sous forme de lignées homozygotes issues directement de la méiose d'hybrides entre parents résistants et sensibles.

La technique d'androgenèse in vitro est maintenant bien maîtrisée chez le piment (DUMAS de VAULX et al., 1981). Nous avons essayé de l'appliquer à l'analyse du support héréditaire de la résistance aux nématodes chez les nouveaux géniteurs : PM 217 et PM 687, lignées par ailleurs utilisées pour d'autres propriétés de résistance à diverses maladies.

\section{MATÉRIEL ET MÉTHODES}

\section{A. Matériel végétal}

Les parents résistants sont 2 lignées de Capsicum annuum L., toutes 2 assez proches du type sauvage, mais extrêmement différentes l'une de l'autre. La lignée PM 217 est tirée de la population PI 201234, originaire d'Amérique Centrale. Elle a été largement utilisée pour sa résistance à Phytophthora capsici Leonian. La lignée PM 687 est tirée de PI 322719 qui proviendrait de l'Inde. Cette lignée possède une résistance partielle à Xanthomonas vesicatoria (Doidge) Dowson et à Leveillula taurica Arnaud.

La résistance de PM 217 concerne la totalité des espèces et souches de nématodes utilisées à ce jour, soit 4 espèces (HENDY et al., 1983). Elle autorise parfois la formation de rares galles, mais celles-ci régressent à la suite de phénomènes de nécrose localisée : il s'agit probablement d'un mécanisı..e d'hypersensibi- lité. La résistance de PM 687 concerne seulement les 3 principales espèces de Meloidogyne : $M$. arenaria, $M$. incognita et $M$. javanica. Elle est totale car elle ne permet pas l'installation des nématodes sur la racine parce que les pénétrations sont rares et entraînent immédiatement des nécroses.

Le parent-hôte opposé à chacune de ces variétés est "Yolo Wonder ». Cette variété, en ce qui concerne ses relations avec les nématodes, n'est pas quelconque, puisqu'elle possède déjà une résistance d'un bon niveau vis-à-vis de $M$. javanica et de certaines souches de $M$. arenaria non utilisées dans ces essais (HENDY et al., 1983).

Pour l'étude de la transmission de ces différentes propriétés, on a prélevé et mis en culture les étamines des hybrides $F_{1}$ : PM $217 \times$ «Yolo Wonder » et PM $687 \times$ «Yolo Wonder ». Les plantes haploïdes androgénétiques obtenues ont été traitées à la colchicine pour obtenir le doublement chromosomique. Les secteurs diploïdes ont été autofécondés et la descendance examinée pour vérifier l'état homozygote. Les tests ont été conduits à la $2^{\mathrm{e}}$ génération d'autofécondation, la diploïdisation à la colchicine étant considérée comme une $1^{\text {re }}$ autofécondation.

\section{B. Populations de Meloidogyne}

On a examiné 25 lignées autodiploïdes dans le croisement PM $217 \times$ "Yolo Wonder » (code de la série : 300 ) et 32 dans PM $687 \times$ "Yolo Wonder» (code de la série : 100).

Les populations utilisées dans les tests ont été choisies aussi différentes que possible d'après l'origine géographique et des données isoenzymatiques. Chacune d'elles peut être considérée comme un clone : le polymorphisme isoenzymatique intra-population y est quasi nul (DALMASSO \& BERGE, 1978). Les populations «Monteux» (France) et «Ain Taoujdate» (Maroc) se rattachent à l'espèce $M$. arenaria, "Calissane » (France) à $M$. incognita et « Abou Dhabi » à $M$. javanica. La population «Séville» (Espagne) appartient à une nouvelle espèce.

\section{Technique d'inoculation et d'évaluation}

Les plantes cultivées en pots de $200 \mathrm{ml}$ reçoivent environ 1000 larves et œufs au stade 6 feuilles apparentes. Pour chaque lignée autodiploïde et chaque population de nématodes on a testé 5 plantes.

L'expérimentation a été conduite dans de petits compartiments de serre, climatisés de façon à ce que la température n'excède pas $30^{\circ} \mathrm{C}$.

Cinq semaines après l'inoculation, le système racinaire de chaque plante a été examiné après lavage et les pontes visibles ont été comptées. Le nombre de pontes par plante varie, chez les parents et témoins, dans la gamme suivante (ensemble des tests) : PM $217=$ 0-11; PM $687=0$; « Yolo Wonder » = 41-109, sauf en présence de $M$. javanica auquel il est résistant : 1-6; «Doux des Landes »: 112-245.

Les descendants des croisements de ces variétés donnant moins de 10 pontes par plante (en général 0 à 3) sont considérés comme résistants ; ceux qui en donnent plus que les parents résistants sont considérés comme hôtes. 


\section{RÉSULTATS}

\section{A. Descendance du croisement PM $217 \times$ « Yolo Wonder » (tabl. 1)}

Les 25 lignées autodiploïdes, haploïdes diploïdes issues d'androgenèse (HDA) obtenues dans ce croisement se classent dans l'une des catégories "résistante » ou « hôte » en présence de l'une des 5 populations de nématodes. Chaque lignée peut être caractérisée par l'ensemble des 5 réponses obtenues. On retrouve, bien entendu, les phénotypes parentaux, mais aussi 3 phénotypes nouveaux et, parmi eux, des lignées sensibles aux 5 populations. Les résultats sont reproduits au tableau $I$.

L'utilisation des lignées HDA élimine le risque provenant de la variabilité naturelle, toujours possible chez les variétés sélectionnées et le caractère homozygote de tous les individus évite ainsi les écarts dus à l'interférence entre les divers loci; de là une plus grande homogénéité dans la réponse.

On peut constater que les propriétés de résistance apportées par chacun des parents se décomposent en 3 types élémentaires ( 2 parentaux, 1 nouveau : c) que l'on peut supposer associés à des gènes particuliers, l'un provenant de "Yolo Wonder ", les 2 autres, à spectre plus étendu, provenant de PM 217.

La résistance de "Yolo Wonder » vis-à-vis de $M$. javanica réapparaît, sans aucune autre, dans 2 HDA (301 et 342). PM 217 ne possède pas d'allèle actif au même locus, puisque l'on trouve 6 HDA sensibles à toutes les souches, y compris $M$. javanica. On peut admettre, à titre d'hypothèse, que "Yolo Won- der " porte un gène particulier à spectre étroit, désigné provisoirement par le symbole Me5. PM 217 porterait un allèle inactif $\mathrm{Me}^{+}$au même locus. Le gène Me 5 contrôlerait la résistance à $M$. javanica .

7 HDA montrent une résistance simultanée à $M$. sp. "Séville » et $M$. javanica $(309,310, \ldots)$, tout en étant sensibles à $M$. arenaria et $M$. incognita. On suppose que ce comportement est associé à un gène particulier : Me2. On remarque que la résistance à "Séville " implique toujours de façon concomitante la résistance à $M$. javanica. Il y a autant de lignées résistantes que de lignées hôtes vis-à-vis de la population de "Séville », cette proportion de 50 p. 100 de lignées résistantes existe dans 2 phénotypes, un de type parental (résistant aux 5 populations : HDA ${ }^{\circ} 313,318 \ldots$ ) et un de type nouveau (résistant seulement à $M$. javanica et « Séville »: HDA n ${ }^{\circ} 309,310 \ldots$ ).

5 HDA montrent une résistance aux 3 espèces, $M$. arenaria, incognita et javanica, mais non à "Séville » $(306,309, \ldots)$. Le gène responsable de ce comportement reçoit la dénomination Me1.

Notre dénomination des gènes est basée sur leur importance relative. Me1 correspond aux 3 grandes espèces de Meloidogyne, Me2 à $M$. javanica et $M$. sp "Séville ", alors que Me5 ne se rencontre que chez "Yolo Wonder " et n'intéresse que $M$. javanica.

Ainsi, les propriétés de résistance de PM 217 résulteraient de l'action d'au moins 2 gènes distincts et non allèles du gène porté par l'autre parent. Ces 2 gènes Me1 et Me2 sont vraisemblablement indépendants, car on trouve à peu près autant de types parentaux ( 5 fois ils apparaissent ensemble) que de types recombinés ( 5 fois Me1 et 7 fois Me2 apparaissent seuls).

TABLEAU 1

Aptitudes pathogènes de 5 populations-clones de Meloidogyne sur 25 lignées autodiploides (HDA), tirées de la $F_{1}$ : PM $217 \times$ «Yolo Wonder》 (série 300). Matérialisation de l'effet des diverses combinaisons de gènes de résistance.

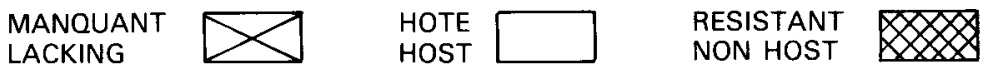

Pathogenicity of 5 clone populations of Meloidogyne spp. on 25 autodiploid lines (HDA) from the $F_{1}$ : $P M 217 \times$ "Yolo Wonder " (series 300). Effect of different combinations of resistance genes.

\begin{tabular}{|c|c|c|c|c|c|c|c|c|c|c|c|c|c|c|c|c|c|c|c|c|c|c|c|c|c|}
\hline \multirow[b]{2}{*}{ Meloidogyne } & $\begin{array}{l}\mathrm{Pa} \text { - } \\
\text { rents }\end{array}$ & \multicolumn{22}{|c|}{ Lignées HDA } & \multirow{2}{*}{$\begin{array}{l}\text { Génotype } \\
\text { supposé }\end{array}$} & \multirow{2}{*}{ Origine } \\
\hline & $\mid \begin{array}{c}1 \\
\sum_{0} \\
0\end{array}$ & s. & (ষ) & 이 & : & 응 & $\left.\frac{m}{m}\right|^{m}$ & $\frac{4}{3}$ & $\frac{5}{n}$ & $\bar{m}$ & $\frac{\infty}{m}$ & $\frac{\sigma}{m}$ & స్లి & ఖ్లి|శ & 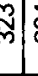 & స్లు & :্লি & స్యి & 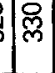 & |్্লি & ষ্ & 憋 & 离 & & \\
\hline $\begin{array}{l}\text { Monteux } \\
\text { M. arenaria }\end{array}$ & $x$ & & $\infty$ & & & & 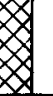 & & & & 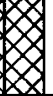 & & & 8 & & & & & 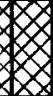 & 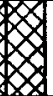 & & 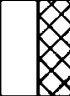 & & $\mathrm{Me}_{1}$ & PM 217 \\
\hline $\begin{array}{c}\text { Ain Taoujdate } \\
M . \text { arenaria }\end{array}$ & $x$ & & $\infty$ & & & & 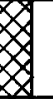 & & & 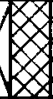 & 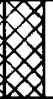 & & & $x$ & & & & & 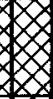 & 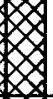 & & 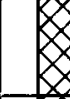 & & $M e_{1}$ & PM 217 \\
\hline $\begin{array}{c}\text { Calissane } \\
M . \text { incognita }\end{array}$ & $\infty$ & & $\infty$ & & & & 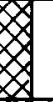 & & & 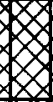 & $\infty$ & & & 8 & & & & & 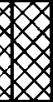 & 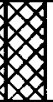 & & 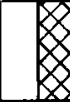 & & $\mathrm{Me}_{1}$ & PM 217 \\
\hline $\begin{array}{l}\text { Abou Dhabi } \\
\text { M. javanica }\end{array}$ & 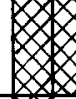 & & $\infty$ & & 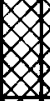 & 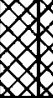 & & & & & & & & 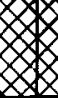 & & & 8 & & 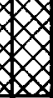 & 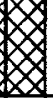 & & $\infty$ & $x$ & $\begin{array}{c}\mathrm{Me}_{5} \\
\mathrm{Me}_{1}, \mathrm{Me}_{2}\end{array}$ & $\begin{array}{l}\text { Yolo Wonder } \\
\text { PM } 217\end{array}$ \\
\hline Séville & $x$ & & & & $\infty$ & & & & 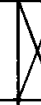 & & & & & $\infty$ & & & 8 & $\infty$ & & & & $x$ & 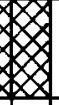 & $\mathrm{Me}_{2}$ & PM 217 \\
\hline $\begin{array}{c}\text { Types } \\
\text { nouveaux }\end{array}$ & & & a & b & c & c & b & t & ? & a & & c & c & c & $c$ & b & c & b & $\mathrm{a}$ & a & b & & c & & \\
\hline
\end{tabular}

* Yolo Wonder 
Si les 3 gènes, Me1, Me2 et Me5 sont situés à des loci indépendants et si l'androgenèse in vitro se produit à la même fréquence sur toutes les combinaisons géniques, on doit voir apparaître 8 génotypes différents et d'égale fréquence, correspondant à une distribution méiotique indépendante. Il n'y a cependant que 5 phénotypes distincts, car la résistance à $M$. javanica peut être contrôlée par chacun des 3 gènes : la présence de Me 5 ne peut pas être décelée si l'un ou l'autre des gènes de PM 217 est déjà présent. Le bilan des observations figure au tableau 2. L'hypothèse de 3 gènes indépendants n'est pas infirmée, bien qu'il y ait un certain excès de lignées hôtes. Cet excès peut se comprendre s'il y a interférence entre un des gènes $\mathrm{Me} 1$ ou Me2 et le gène Me5, c'està-dire dans un certain degré de liaison non allélique, puisqu'il y a des lignées sensibles. Si on fait une place à part pour Me5, on remarque que Mel et Me2 semblent se comporter en gènes à disjonction indépendante.

\section{B. Descendance du croisement PM $687 \times$ «Yolo Wonder " (tabl. 2)}

Pour toutes les combinaisons de souches et de lignées on a, par mesure de simplification, considéré seulement 2 catégories de réponse : "résistante » ou "hôte ». Les propriétés de résistance de 32 lignées HDA sont reportées au tableau 3.

Il apparaît 5 groupes phénotypiques : les types parentaux et 3 phénotypes nouveaux. Des 4 phénotypes résistants, 2 se rapportent aux parents et 2 sont nouveaux. Il y a donc une similitude avec les résultats obtenus dans l'autre croisement. Cependant, le spectre d'efficacité de ces résistances élémentaires est bien différent, à l'exception du caractère apporté par le parent commun, "Yolo Wonder" .

Le gène de résistance porté par "Yolo Wonder» (Me5 actif sur $M$. javanica) apparaît à nouveau (HDA 108, 116, 136, ...). PM 687 doit porter, comme c'était le cas chez PM 217, un allèle inactif $\mathrm{Me}^{+}$, puisqu'il existe des HDA hôtes de $M$. javanica, alors que les 2 parents sont résistants.

La résistance de PM 687 se décompose en 2 éléments distincts correspondant vraisemblablement à 2 gènes distincts. L'un concerne les 3 principales espèces de Meloidogyne, à l'exception de $M$. arenaria «Ain Taoujdate » (HDA 119, 120, 149). Sa dénomination provisoire est Me3. L'autre n'est actif que sur cette seule souche (gène Me4).

La transmission de Me3 par la méiose mâle, puis par la voie androgène, semble suivre la répartition 1 : 1. Il y a, en effet, exactement le même nombre de lignées HDA porteuses que de lignées possédant l'allèle inactif issu du parent sensible (16).

Toutes les HDA, à l'image des parents, sont sensibles à $M$. sp. « Séville ».

Le tableau 4 récapitule l'ensemble des résultats en omettant les 5 lignées qui n'ont pu recevoir la totalité des tests.

Il apparaît que Me3 et Me4 ne sont pas indépendants (probabilité 0,001). Le taux de recombinaison est estimé à $5 / 27$ soit 18,5 p. 100 (limites $6,6-35,1$ p. 100).

\section{Etude du croisement PM $217 \times$ PM 687}

Pour tester l'éventuel allélisme des gènes principaux, portés par chacun des parents (Mel et Me3), on a étudié les disjonctions au niveau de la génération $F_{2}$ du croisement entre ces parents, l'androgenèse n'ayant pas été appliquée. En présence d'une souche de $M$. incognita contrôlée par l'un et l'autre gène, la $F_{2}$ ne doit comporter que des plantes résistantes, si ces 2 gènes sont allèles. S'ils sont distincts et non liés, on doit observer $1 / 16$ de plantes hôtes ces gènes étant dominants, les gènes en question étant fixés pour les 2 piments.

C'est une situation intermédiaire qui apparaît (tabl. 5).

TABLEAU 2

Répartition des lignées HDA tirées de la $F_{1}: P M 217 \times$ «olo Wonder ": regroupement des catégories phénotypiques et génotypes supposés. Test de l'hypothèse d'une disjonction indépendante de 3 gènes dont le spectre d'action se recouvre partiellement $\chi^{2}=3,669$; prob. $=0,30-0,50 . R$ : résistant; $S:$ sensible.

Distribution of the HDA lines obtained from the F, PM $2 I 7 \times$ "Yolo Wonder" into phenotypic and hypothetical genotypic categories. Test of the hypothesis : independent segregation of 3 genes with partially overlapping activities. $\chi^{2}=3.669 ;$ prob. $=0.30-0.50 . R:$ resistant $;$ S: susceptible.

\begin{tabular}{|c|c|c|c|c|c|c|c|}
\hline $\begin{array}{c}\text { Référence } \\
\text { des lignées HDA }\end{array}$ & $\begin{array}{l}M . \text { arenaria } \\
M . \text { incognita }\end{array}$ & M. javanica & $\begin{array}{l}M . \text { sp. } \\
\text { Séville }\end{array}$ & $\begin{array}{l}\text { Génotype } \\
\text { supposé }\end{array}$ & $\begin{array}{l}\text { Proportions } \\
\text { attendues }\end{array}$ & $\begin{array}{l}\text { Fréqu } \\
\text { Obs. }\end{array}$ & $\begin{array}{l}\text { ences } \\
\text { Calc. }\end{array}$ \\
\hline $313,318,322,328,347$ & $\mathrm{R}$ & $\mathrm{R}$ & $\mathbf{R}$ & $\mathrm{Me} 1, \mathrm{Me} 2, \underset{+}{\mathrm{Me} 5}$ & $2 / 8$ & 5 & 6,25 \\
\hline $306,316,317,330,336$ & $\mathrm{R}$ & $\mathrm{R}$ & $\mathrm{S}$ & $\mathrm{Mel},+, \begin{array}{c}\text { Mes } \\
+\end{array}$ & $2 / 8$ & 5 & 6,25 \\
\hline $309,310,319,323,326,327,348$ & $\mathrm{~S}$ & $\mathrm{R}$ & $\mathbf{R}$ & $+, \operatorname{Me} 2, \stackrel{\text { Me5 }}{+}$ & $2 / 8$ & 7 & 6,25 \\
\hline 301,342 & S & $\mathrm{R}$ & $\mathrm{S}$ &,,$++ \operatorname{Mes}$ & $1 / 8$ & 2 & 3,125 \\
\hline $307,314,315,320,324,341$ & $\mathrm{~S}$ & $\mathrm{~S}$ & $\mathrm{~S}$ &,,+++ & $1 / 8$ & 6 & 3,125 \\
\hline
\end{tabular}


TABLEAU 3

Aptitudes pathogènes de 5 populations de Meloidogyne spp. sur 32 lignées HDA tirées de la $F_{1}: P M 687 \times$ "Yolo Wonder " (série 100): matérialisation de l'effet des combinaisons de gènes de résistance.

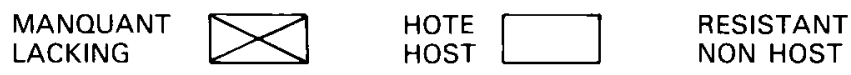

Pathogenicity of 5 clone populations of Meloidogyne spp. on 32 HDA lines from the $F_{7}: P M 687 \times$ "Yolo Wonder " (series 300$)$. Effect of different combinations of resistance genes.

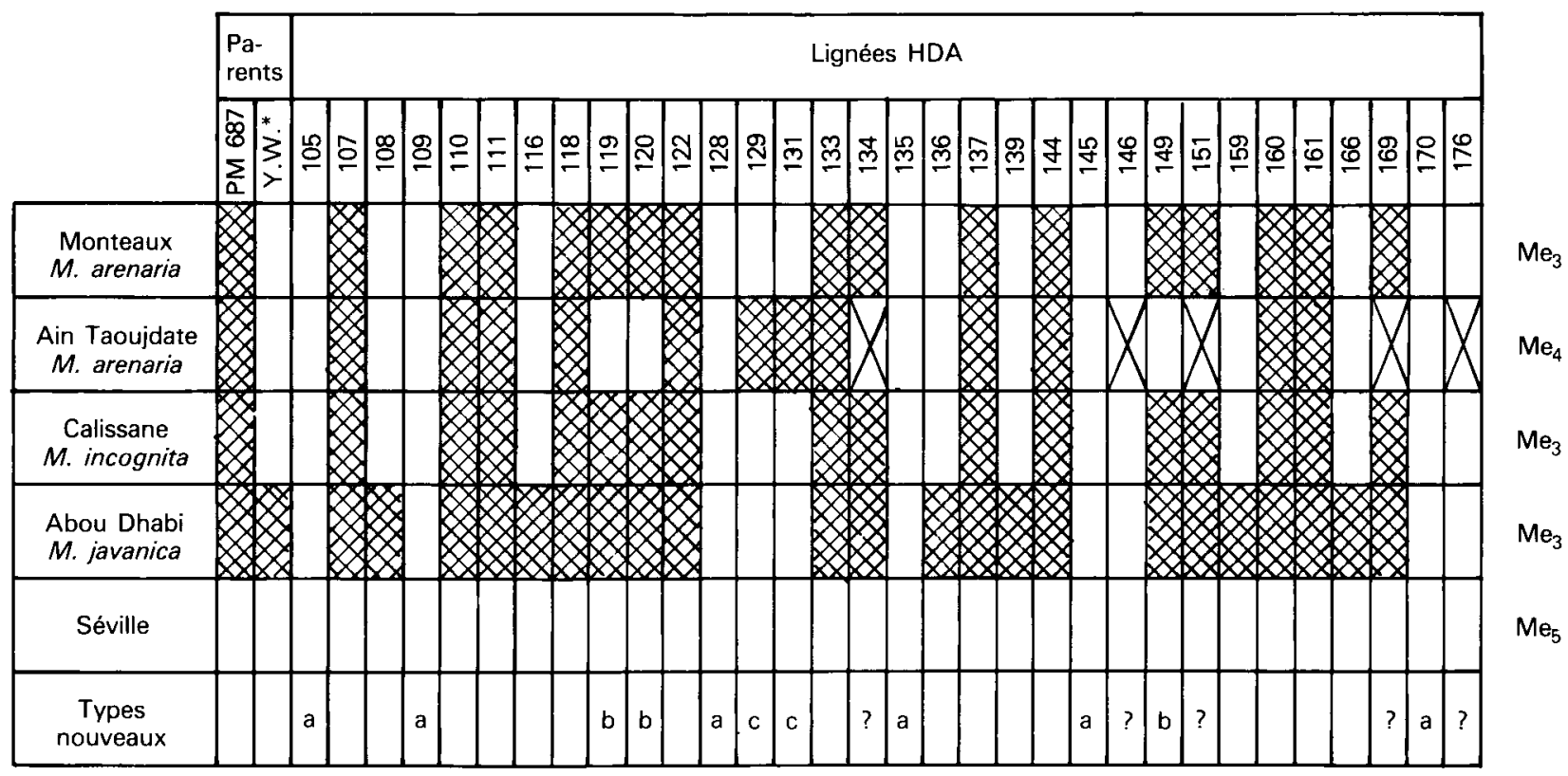

* Yolo Wonder

TABLEAU 4

Répartition des lignées HDA tirées de la $F_{1}: P M 687 \times$ «Yolo Wonder 》. Regroupement par catégories phénotypiques et génotypes supposés. 26 lignées opposées à 5 populations clones de Meloidogyne. Test de l'hypothèse d'une disjonction indépendante des gènes Me3 et Me4. $\chi^{2}=11,07$. Prob. $<0,02$ (test $\left.1: 1: 1: 1\right) ; \chi^{2}=10,70$ (test 1 type parental $: 1$ type recombiné).

Distribution of the HDA lines obtained from the F, PM $687 \times$ «Yolo Wonder into phenotypic and hypothetical genotypic categories. 26 lines inoculated with five clone populations of Meloidogyne. Test of the hypothesis : independent segregation of the two genes Me3 and Me4. $\chi^{2}=11.07$. Prob. $<0.02$ (test $\left.1: 1: 1: 1\right) ; \chi^{2}=10.70$ (test 1 parental $: 1$ recombined).

\begin{tabular}{|c|c|c|c|c|c|c|c|}
\hline $\begin{array}{c}\text { Référence } \\
\text { des lignées HDA }\end{array}$ & $\begin{array}{l}M . \text { arenaria } \\
M . \text { incognita }\end{array}$ & M. javanica & $\begin{array}{l}M . \text { sp. } \\
\text { Séville }\end{array}$ & $\begin{array}{l}\text { Génotype } \\
\text { supposé }\end{array}$ & $\begin{array}{l}\text { Proportions } \\
\text { attendues }\end{array}$ & $\begin{array}{l}\text { Fréqu } \\
\text { Obs. }\end{array}$ & $\begin{array}{l}\text { ences } \\
\text { Calc. }\end{array}$ \\
\hline $\begin{array}{l}107,110,111,118,122, \\
133,137,144,160,161\end{array}$ & $\mathrm{R}$ & $\mathrm{R}$ & $\mathbf{R}$ & $\begin{array}{c}\text { Me3, Me4, Me5 } \\
\text { Me3 Me4 }+\end{array}$ & $1 / 4$ & $10(p)$ & 6,75 \\
\hline $119,120,149$ & $\mathrm{R}$ & $\mathrm{S}$ & $\mathrm{R}$ & $\begin{array}{l}\mathrm{Me} 3+\mathrm{Me} 5 \\
\mathrm{Me} 3++\end{array}$ & $1 / 4$ & $3(r)$ & 6,75 \\
\hline 129,131 & S & $\mathbf{R}$ & $\begin{array}{l}\mathbf{R} \\
\mathbf{S}\end{array}$ & $\begin{array}{l}+\mathrm{Me} 4 \mathrm{Me} 5 \\
+\mathrm{Me} 4+\end{array}$ & $1 / 4$ & $2(r)$ & 6,75 \\
\hline $\begin{array}{l}108,116,136,139,159,166 \\
105,109,128,135,145,170\end{array}$ & $S$ & S & $\begin{array}{l}\mathrm{R} \\
\mathrm{S}\end{array}$ & $\begin{array}{l}++\operatorname{Me} 5 \\
+++\end{array}$ & $1 / 4$ & $12(p)$ & 6,75 \\
\hline
\end{tabular}

On observe des plantes hôtes mais dans des proportions inférieures à $1 / 16$, soit $2 / 127\left(\chi^{2}=4,74\right.$; prob. $<0,05)$.

Il est donc probable que Me1 et Me3 sont liés. Le taux de recombinaison estimé est de l'ordre de
25 p. $100\left(\mathrm{p}^{2} / 4=1,57\right.$ p. 100$)$.

La disjonction relative au gène Me2 révélé par son effet sur $M$. sp. "Séville 》 est conforme à la distribution mendélienne $3: 1$, ce gène étant, lui aussi, dominant $\left(\chi^{2}=1,31\right.$; prob. $\left.0,20-0,30\right)$. 
TABLEAU 5

Etude des $I^{r e}$ et $2^{e}$ générations du croisement PM $217 \times P M 687$ : répartition des plantes pour leur résistance à 2 souches de Meloidogyne : l'une n'attaquant aucun des parents (M. incognita, Calissane), l'autre attaquant le seul parent PM 687 (M. sp., Séville).

Study of $F_{1}$ and $F_{2}$ progenies from the cross $P M 217 \times P M 687$ : distribution of the plants according to their resistance to 2 Meloidogyne populations: one, M. incognita (Calissane) being controlled by both parents, the other, M. sp. (Seville) being aggressive to PM 687.

\begin{tabular}{|c|c|c|c|c|}
\hline \multirow{3}{*}{ Parents, $\mathbf{F}_{1}, \mathrm{~F}_{2}$} & \multicolumn{2}{|c|}{ M. incognita «Calissane » } & \multicolumn{2}{|c|}{ Meloidogyne sp. «Séville» } \\
\hline & \multicolumn{2}{|c|}{ Nombre de plantes } & \multicolumn{2}{|c|}{ Nombre de plantes } \\
\hline & Résistantes & Multiplicatrices & Résistantes & Multiplicatrices \\
\hline PM 217 & 29 & 0 & 21 & 0 \\
\hline PM 687 & 21 & 0 & 0 & 22 \\
\hline $\mathrm{F}_{1}(\mathrm{PM} 217 \times \mathrm{PM} 687)$ & 31 & 0 & 34 & 0 \\
\hline $\mathrm{F}_{2}(\mathrm{PM} 217 \times \mathrm{PM} 687)$ & 125 & 2 & 107 & 28 \\
\hline
\end{tabular}

\section{DISCUSSION - CONCLUSIONS}

Les résistances portées sur les 3 parents étudiés sont contrôlées par un nombre de gènes qui ne peut être inférieur à 5 . On a attribué des symboles provisoires à chacun de ces gènes en attendant de pouvoir conduire les tests d'allélisme avec le gène $\mathrm{N}$ de HARE (1957).

Il apparaît que 3 de ces gènes sont liés de façon assez lâche, Me1, Me3 et Me4. Par contre, Me2, provenant de PM 217, ne fait pas partie de ce groupe de linkage, pas plus que Me5 porté par "Yolo-Wonder ».

On pourrait classer de façon encore plus précise ces gènes en fonction de l'étendue de leur spectre d'action et, secondairement, du mode d'expression au niveau des racines (non-pénétration ou non-installation des nématodes, apparition d'un petit nombre de galles tendant à se nécroser ou non, production ou non de capsidiol...); de telles études sont actuellement en cours.

Dans la gamme d'hôtes différentiels, établie par SASSER (1979), figure la variété "California Wonder » (dont est issu « Yolo Wonder »), donnée pour résistante à $M$. javanica et à certaines souches de $M$. arenaria. D'après nos propres résultats (HENDY et al., 1983), "Yolo Wonder » a hérité des mêmes propriétés. Il serait intéressant de savoir si le gène Me5 est responsable de ces 2 types de résistance. Les lignées HDA offrent, pour tester cette hypothèse, un matériel de choix.

D'après nos résultats, Me4 aurait un spectre très étroit, ne contrôlant qu'une souche de $M$. arenaria. Me2 contrôle à la fois $M$. sp. "Séville " et $M$. javanica. Me3 est responsable de l'absence de développement des larves des 3 principales espèces sur les racines de piment. Cet effet est cependant inefficace en présence de certaines souches de $M$. arenaria. Me1, enfin, semble capable de contrôler toutes les souches des 3 mêmes espèces, par un mécanisme impliquant la formation de réactions nécrotiques, plus ou moins rapides, après l'installation des larves.
Si l'on considère le nombre de gènes capables de contrôler les différentes espèces, on constate que 3 sont actifs sur $M$. javanica, 2 sur $M$. arenaria et $M$. incognita, un seul sur $M$. sp. "Séville ".

On remarque que pour Me1 la proportion de chacun des types parentaux (tabl. 1) n'est pas parfaitement de $1: 1$.

Di VITO \& SACCARDO (1978) ont trouvé des résistances à $M$. incognita chez Capsicum chinense Jacq. et Capsicum frutescens $\mathrm{Bl}$. ; on ne dispose pas encore d'informations détaillées sur la nature des gènes qui contrôlent ces propriétés. Il sera intéressant de préciser si ces gènes sont distincts de ceux que nous avons identifiés chez $C$. annuum.

Chez la tomate, on utilise très largement une résistance de type hypersensibilité, trouvée en 1944 chez Lycopersicon peruvianum (L.) Mill. et transférée à la tomate cultivée (GILBERT \& MC GUIRE, 1956). On considère généralement que cette résistance est liée à un gène dominant Mi. Cependant, SIDHU \& WEBSTER (1975) affirment qu'il s'agit d'un ensemble de 3 gènes étroitement liés. Il reste à caractériser, de façon précise, l'effet de chacun d'entre eux. Quoi qu'il en soit, cette résistance est inefficace en présence de certaines populations de nématodes, par exemple $M$. arenaria "Le Grau », ou $M$. incognita «Valbonne ", isolées dans le Sud de la France. Les géniteurs de piment PM 217 et PM 687 sont résistants à ces 2 populations et peuvent donc concourir à leur contrôle (HENDY et al., 1983).

D'une manière plus générale, l'existence de lignées homozygotes de piment possédant l'un ou l'autre des 5 gènes identifiés permettra de caractériser plus finement les propriétés pathogènes des populations de nématodes et les divers mécanismes de résistance qui leur sont respectivement associés.

Reçu le 19 avril 1984. Accepté le 7 septembre 1984 . 


\section{RÉFÉRENCES BIBLIOGRAPHIQUES}

Dalmasso A., Bergé J. B., 1978. Molecular polymorphism and phylogenetic relationships in some Meloidogyne spp. : application to the taxonomy of Meloidogyne. J. Nematol., 10, 324-332.

Di Vito M., Saccardo F., 1978. Riposta di linee e varieta di Capsicum spp. agli attachi di Meloidogyne incognita in serra. Nematol. Mediter., 6, 83-88.

Dumas de Vaulx R., Chambonnet D., Pochard E., 1981. Culture in vitro d'anthères de piment (Capsicum annuum L.) : amélioration des taux d'obtention des plantes chez différents génotypes par des traitements à $+35^{\circ} \mathrm{C}$. Agronomie, 1, 859-864.

Gilbert J. C., Mc Guire D. C., 1956. Inheritance of resistance to severe root-knot from Meloidogyne incognita in commercial type tomatoes. Proc. Am. Soc. Hortic. Sci., 68, 437-442.

Hare W. W., 1956. Resistance in pepper to Meloidogyne incognita acrita. Phytopathology, 46, 98-104.
Hare W. W., 1957. Inheritance of resistance to root-knot nematodes in pepper. Phytopathology, 47, 455-459.

Hendy H., Pochard E., Dalmasso A., 1983. Identification de deux nouvelles sources de résistance aux nématodes du genre Meloidogyne chez le piment, Capsicum annuum L. C. R. Acad. Agric. Fr., 817-822.

Sasser J. N., 1979. Pathogenicity host ranges and variability in $M$. species, 257-268. In Lamberti F., Taylor C. E. : "Root-knot nematodes (Meloidogyne species) ; systematics, biology and control $\gg$. Acad. Press, London, 477 p.

Sidhu G. S., Webster M., 1975. Linkage and allelic relationships among genes for resistance in tomato (Lycopersicon esculentum) against Meloidogyne incognita. Can. J. Genet. Cytol., 17, 323-328. 